\section{Continuity of care for patients with schizophrenia in commu- nities with limited resources}

\section{Andy Trang} Department of Psychiatry, UC Irvine
School of Medicine, Irvine, CA, USA

For psychiatric patients with schizophrenia the rate of readmission to the hospital can be high and the need for continuation of quality care after hospitalization is tremendous and plays a large role in helping patients remain stable to prevent readmission. However, such resources for continuing care are often difficult to obtain, especially in rural communities where access to such services are limited and the burden of care is frequently placed on family members who do not have formal training in caring for patients with such psychiatric illnesses. In such communities, it is important to emphasize education for the caregivers and establishing a system of support from local community organizations for both the patient and their caregivers to reduce caregiver burnout as well as prevent rehospitalization for the patient.

Studies by Juntapim et al. evaluated such continuity of care in rural Thailand and emphasized the need for community involvement including family, neighbors, and community leaders for the continuing care of the patient's mental illness as well as social support.
While such community support is invaluable, it often places a large burden on caregivers to provide such social, emotional, and financial support. A meta-analysis by Chen et al. demonstrated that non-pharmacological interventions such as psychoeducation, mutual support, and interventions through counseling, telephone, and the internet for such caregivers was able to improve quality of life without changing family functioning, support, and overall satisfaction. ${ }^{1}$ In addition to the range of burdens placed on the caregiver in continuing to care for patient's with schizophrenia, they are also often subjected to violence from the patients and may have fatal consequences, as reported by Kageyama et al. ${ }^{2}$

The need for patients with schizophrenia to have increased access to continuing care resources after hospitalization remains high and the burden of care is often placed on family members with limited training and resources. Despite such limited resources in rural communities, improvements on quality continuity of care is still possible by emphasizing education for caregivers in the community such as family members, recruiting support from local organizations such as religious centers, and having a clear crisis plan in the event that the patient psychiatric condition worsens. Additionally, intervention such as education, counseling, and community support for caregivers may help to reduce the burden of care and improve quality of life for both the caregiver and the patient.
Correspondence: Andy Trang, Department of Psychiatry, UC Irvine School of Medicine, Irvine, CA, USA

E-mail: atrang2@uci.edu

Key words: schizophrenia, continuity of care, limited resources community.

Conflict of interest: the author declares no potential conflict of interest

Funding: none.

Received for publication: 18 January 2018. Accepted for publication: 18 January 2018.

This work is licensed under a Creative Commons Attribution-NonCommercial 4.0 International License (CC BY-NC 4.0).

(C) Copyright A. Trang, 2018

Licensee PAGEPress, Italy

Mental Illness 2018; 10:7585

doi:10.4081/mi.2018.7585

\section{References}

1. Hsu TJ, Tsai HT, Hwang AC, et al. Predictors of non-pharmacological intervention effect on cognitive function and behavioral and psychological symptoms of older people with dementia. Geriatr Gerontol Int 2017;17:28-35.

2. Kageyama M, Yokoyama K, Nagata S, et al. Rate of family violence among patients with schizophrenia in Japan. Asia-Pacific J Publ Health 2015;27: 652-60. 